\title{
25 Research Square \\ Behavioural and Physiological Response to \\ Frustration in Autistic Youth: Associations with Irritability
}

Virginia Carter Leno ( $\square$ virginia.carter_leno@kcl.ac.uk)

King's College London https://orcid.org/0000-0002-7455-5514

Georgia Forth

King's College London

Susie Chandler

King's College London

Philippa White

King's College London

Isabel Yorke

King's College

Tony Charman

King's College London

Andrew Pickles

King's College London

Emily Simonoff

King's College London

\section{Research}

Keywords: irritability, frustration, physiology, autism spectrum disorders, heart rate

Posted Date: October 26th, 2020

DOI: https://doi.org/10.21203/rs.3.rs-96188/v1

License: (1) (1) This work is licensed under a Creative Commons Attribution 4.0 International License.

Read Full License

Version of Record: A version of this preprint was published at Journal of Neurodevelopmental Disorders on July 19th, 2021. See the published version at https://doi.org/10.1186/s11689-021-09374-1. 


\section{Abstract}

Background: Irritability is a common and impairing occurrence in autistic youth, yet the underlying mechanisms are not well known. In typically developing populations, differences in frustration response have been suggested as important driver of the behavioural symptoms of irritability. Research exploring the role of frustration response as a risk factor for irritability in autistic populations is limited and often uses on parent report or observer ratings; objective measures of frustration response appropriate for use in autistic populations are required to advance the field.

Methods: In the current study, fifty-two autistic adolescents aged 13-17 years from a population-based longitudinal study completed an experimental task designed to induce frustration through exposure to periods of unexpected delay. Behavioural (number of button presses) and physiological (heart rate; HR) metrics were collected during delay periods. Irritability was measured using the parent-rated Affective Reactivity Index (ARI). Analyses used mixed-level models to test whether irritability was associated with different slopes of behavioural and physiological response in response to experimentally induced frustration. Age and baseline HR (for the physiological data only) were included as covariates.

Results: Analyses showed a marginal association between irritability and the slope of behavioural response (incident rate ratio $=.98, \mathrm{p}=.06$ ), and a significant association with the slope of physiological response $(b=-.10, p=.04)$; higher levels of irritability were associated with a dampened behavioural and physiological response, as indicated by flatter slopes of change over the course of the task. This pattern of results remained when adjusting for IQ, autism symptom severity and medication use (association between irritability and slope of behavioural response: incident rate ratio $=.98, p=.02$; slope of HR response; $b=-.10, p=.04)$.

Conclusions: Results suggest that the current experimental task may be a useful objective measure of frustration response for use with autistic populations, and that a non-adaptive response to frustration may be one biological mechanism underpinning irritability in autistic youth. This may represent an important target for future intervention studies.

\section{Introduction}

Irritability is a trans-diagnostic concept defined as "inter-individual differences in proneness to anger that may reach a pathological extent" (1). Pathological irritability can have an extremely negative impact on a young person's education, home life and well-being; in typically developing populations (we use this term to refer to populations without developmental disorders such as autism spectrum disorder) it is associated with increased likelihood of future depression, anxiety and oppositional defiant disorder, and poorer life outcomes (e.g., lower income level and worse physical health) (1). Within the most recent diagnostic manual, pathological levels of irritability form a major part of the diagnostic criteria for disruptive mood dysregulation disorder (DMDD; 2); this was previously captured by the syndrome of severe mood dysregulation (3). It should also be noted irritability is not specific to DMDD, it is also listed 
as symptom of depression and post-traumatic stress disorder, and is closely related to the 'touchy and easily annoyed' symptom of oppositional defiant disorder (2).

Autistic youth (the term autistic will be used throughout in keeping with stakeholder preferences), characterised by impairments in social communication abilities and the presence of restricted and repetitive behaviours and interests and sensory differences (2) are found to have elevated rates of irritability (4-6). In autistic populations, high levels of irritability often manifest as behavioural problems such as oppositional behaviour, aggression, temper tantrums and severe non-compliance $(7,8)$. Currently, the mechanisms underpinning variation in irritability in both typically developing and autistic populations are not well understood. Better aetiological understanding will pave the way for more targeted interventions, and given the reported negative outcomes associated with irritability, promote positive outcomes in autistic youth.

\section{Experimental Correlates of Irritability in Typically Developing Youth}

Previous work in typically developing children on the cognitive and neural correlates of irritability has primarily implicated alterations in emotion processing and aberrant response to frustration $(9,10)$. Aberrant responses to frustration are often studied using laboratory-based tasks that either delay or block goal attainment, normally rewards. Typically developing children with severe irritability (operationalized as meeting criteria for severe mood dysregulation) rate themselves as being more aroused than children without irritability (but no different from children with bipolar disorder) in response to experimentally induced frustration (11). Typically developing children with severe irritability also show poorer performance on spatial attention tasks, and less neural activation to negative feedback in the parietal, parahippocampal, and thalamic/cingulate/striatal regions, during conditions of experimentally induced frustration (12). In young children, higher levels of irritability are associated with decreased activation in the anterior cingulate and striatum during conditions of frustration (13). As the research base of studies of frustration and irritability is still relatively limited, one can also look to research with related behavioural phenotypes with high levels of irritability, for example disruptive behaviour disorders (characterised by aggression, rule-breaking and non-compliance/oppositionality), although it should be noted that irritability is more characteristic of oppositional defiant disorder as compared to conduct disorder. Children with disruptive behaviour disorders (i.e., with a diagnosis of oppositional defiant or conduct disorder) exhibit a blunted physiological response, as indicated by changes in heart rate (HR) or electrodermal activity, to frustration and stress $(14,15)$. Furthermore, in youth with disruptive behaviour disorders, higher stress reactivity predicted decline in aggressive behaviour one year later (16). Similarly, adults from the general population with high levels of trait anger also show decreased neural response to experimentally induced frustration (17). However, meta-analyses find that overall, children and adolescents with conduct problems are characterised by increased HR reactivity (18). Heterogeneity in the directionality of results may in part be due to differing proportions of youth with conduct vs. oppositional defiant disorder across different samples, and therefore variable levels of irritability. 


\section{Correlates of Irritability in Autistic Youth}

Despite the high prevalence of irritability in autistic populations, limited work exists to address underpinning mechanisms, and understand whether the experimental correlates are comparable to those reported from non-autistic (e.g. typically developing) populations. One study found irritability predicted physiological (cortisol and HR) response to stress in autistic youth (19), in that autistic youth with high levels of irritability had a blunted physiological stress response, although results became non-significant when adjusting for levels of anxiety. However, this work focused on response to stress, rather than frustration. In typically developing adults, fear vs. anger in response to stress are associated with distinct biological profiles (20), thus responses to frustration may be a clearer correlate of irritability (as compared to anxiety) in autistic youth. Although irritability was not directly measured, another recent study found no association between observer-rated response to frustration and behavioural problems in autistic children (21). However, the lack of association may have been due in part to limited variance in behavioural problems, as the sample consisted of individuals who had been hospitalized due to severe psychiatric difficulties. Conversely, others find that increases in peak HR moderately predict subsequent episodes of challenging behaviour in autistic children aged 2-4 years (22). In older autistic children (aged 4-7 years), parent ratings of poorer emotion regulation predicted both higher levels of concurrent behavioural problems, and a worsening of behaviour problems at follow up one year later (23).

\section{Aims}

The current paper tests the association between response to frustration and irritability in a populationbased sample of autistic youth. Reviews of studies of emotional responsivity in autistic populations note the over-reliance on parent or self-report measures (unlike in typically developing populations, where experimental measures are better developed) (24), highlighting the need for objective measures of emotional response and regulation suitable for use in autistic youth. We adapt an existing experimental task designed to evoke frustration, which captures both behavioural and physiological responses, to comprehensively capture individual variation in frustration response. Based on the existing literature, we hypothesised that irritability would be associated with a greater behavioural and physiological response to frustration.

\section{Method}

\section{Sample}

Participants were part of the QUEST follow-up study (25), a longitudinal population-based sample recruited at age $4-8$ years (Wave $1 ; N=277$ ) and followed up at ages $11-15$ years (Wave $2 ; N=211$ ) and 13-17 years (Wave 3; $\mathrm{N}=214$ ), as part of the wider IAMHealth project. The original target population for the study was all children born in a four-year period, living in two London boroughs, who had a clinical diagnosis of ASD. 277 children were recruited into the study upon entry and selectively stratified into an 'intensively studied' (hereafter intensive; $n=101$ ) and 'extensively studied' group (hereafter extensive; $n=$ 176) and this sampling structure was maintained at subsequent waves of data collection. The current 
study focuses on the Wave 3 intensive group only. Although all participants had a clinical diagnosis of autism spectrum disorder, the intensive group had their diagnosis confirmed at Wave 2 with the Autism Diagnostic Observation Schedule-2 (ADOS-2; 26), and a subset also with the Autism Diagnostic InterviewRevised (ADI-R; 27). All participating families gave their written informed consent (from young people themselves if $\geq 16$ years in age and were deemed to have capacity, otherwise from parents or caregivers) and the study was approved by Camden and King's Cross Ethics Sub-Committee (17/LO/2098 for Wave $2,17 /$ LO/0397 for Wave 3). Table 1 gives a comparison of key measures between the full Wave 3 intensive sample $(n=77)$ versus the Wave 3 intensive subsample who completed the experimental frustration task $(n=52)$.

Table 1

Wave 3 Full Sample and Subsample Demographic Information.

\begin{tabular}{|c|c|c|c|}
\hline $\begin{array}{l}\text { Mean (standard } \\
\text { deviation; range) }\end{array}$ & $\begin{array}{l}\text { Full Intensive } \\
\text { Sample }(n=77)\end{array}$ & $\begin{array}{l}\text { Sample Who Completed } \\
\text { Frustration Task }(n=52)\end{array}$ & $\begin{array}{l}\text { t-test of group } \\
\text { differences }\end{array}$ \\
\hline \multirow[t]{2}{*}{ Age } & 15.38 & 15.40 & \multirow[t]{2}{*}{$p=.61$} \\
\hline & $(1.16 ; 13.2-17.8)$ & $(1.10 ; 13.2-17.3)$ & \\
\hline$\%$ male $(n)$ & $60 \%(46)$ & $63 \%(33)$ & $p=.61$ \\
\hline \multirow[t]{2}{*}{$\mathrm{IQ}^{\wedge}$} & 69.88 & 84.54 & \multirow[t]{2}{*}{$\mathrm{p}<.001$} \\
\hline & $(31.36 ; 19-129)$ & $(21.74 ; 33-129)$ & \\
\hline \multirow{2}{*}{$\begin{array}{l}\text { Autism Severity^ } \\
\text { (ADOS-CSS) }\end{array}$} & 6.72 & 6.31 & \multirow[t]{2}{*}{$p=.32$} \\
\hline & $(2.66 ; 1-10)$ & $(2.83 ; 1-10)$ & \\
\hline \multirow[t]{2}{*}{ ARI Total } & 3.74 & 4.02 & \multirow[t]{2}{*}{$p=.56$} \\
\hline & $(3.26 ; 0-12)$ & $(3.37 ; 0-12)$ & \\
\hline
\end{tabular}

\section{Measures}

\section{Psychiatric Symptoms}

\section{Clinical Interview}

The Child and Adolescent Psychiatric Assessment-parent version (CAPA; 28, 29) is an interviewer-based structured diagnostic interview for use with children aged $9-17$ years. This was used to identify symptoms of psychiatric disorders that had been present in the past three months. The current study 
uses the total count of oppositional defiant disorder (ODD) symptoms (aside from the 'spiteful/vindictive behaviour' and 'blames others' items as these had $<5$ endorsements across the whole sample).

\section{Parent-Rated Questionnaires}

\section{Affective Reactivity Index (ARI)}

The ARI (30) was used to assess participants' level of irritability and includes six items relating to feelings/behaviours specific for irritability and one question assessing impairment due to irritability, with a higher score indicative of a higher level of irritability. The internal consistency was examined in the full QUEST sample (Wave 2 intensive + extensive; $n=201$ ) and found to be excellent ( $a=0.90)$, and comparable to that reported previously in samples of autistic young people $(a=0.82)(19)$.

\section{Aberrant Behavior Checklist (ABC) - Irritability subscale}

The $A B C$ (31) is a measure developed to assess behaviour problems in children with developmental and intellectual disabilities. The 15-item Irritability subscale used currently is often used as an outcome measure in clinical trials (32). The internal consistency of the subscale was examined in the current intensive sample and found to be excellent $(a=0.93)$.

\section{Direct Assessments}

\section{Baseline HR}

Prior to beginning the task battery, participants watched relaxing videos for five minutes to obtain an estimate of their baseline HR. Average HR was calculated across the four consecutive 30 second segments of data collected during this period, the first 60 seconds and the last 120 seconds were excluded to ensure data quality.

\section{Frustration Task}

A novel task was designed and programmed in E-Prime 2.0 (33), based on a previously described delay frustration task $(34,35)$. The task was simplified to allow maximum participation in our sample. Participants were asked to select the smallest square from a choice of three. To motivate participation, participants were informed that most people their age completed around 60 trials, and a pie was shown for each trial to indicate how much time they left (see Figure 1 for a schematic of the task). However, during the task participants experienced several unexpected delays ( 14 delay trials out of a total of 50 trials), where the computer became unresponsive to their button presses for six seconds. These were pseudo-randomly presented, in that the first six trials were always non-delay trials, and the order of presentation was the same for each participant. The number of button presses was recorded during each six-second delay trial. The task lasted approximately five minutes and was part of a wider task battery.

\section{Physiological Data Extraction and Processing}


Electrocardiogram (ECG) data were recorded at 2000Hz using BIOPAC systems MP160 with BioNomadix wireless transmitters. Data was collected and processed using AcqKnowledge 5.0.1 (36). ECG measurements were collected using electrodes placed in the lead-II position on the back. The ECG signal was filtered using a Comb Band $50 \mathrm{~Hz}$ filter to remove electrical noise and a $1 \mathrm{~Hz}$ High Pass filter to remove baseline drift and movement artefact. $\mathrm{R}$ wave peaks, each representing a heartbeat, were automatically identified and labelled using the AcqKnowledge find cycle protocol. The signal was visually inspected to ensure that $\mathrm{R}$ wave peaks had been correctly identified and any movement artefact removed. HR was extracted for each inter-beat interval during the six-second delay periods. Digital markers indicating the beginning and end of each delay trial were sent via E-Prime, and these were used to demarcate the segments of data extraction.

For both the baseline and experimental task recording, four participants from the 52 who completed the direct assessments had no usable ECG data due to electrode refusal $(n=2)$ and corrupted data files $(n=2)$. For the baseline recording, ECG segments with more than three consecutive missing peaks or $10 \%$ of data missing were excluded (as in previous studies of autistic populations) (37), and participants with $\geq 50 \%$ missing task data overall were excluded $(n=4)$, leaving a final sample of $n=44$ for the baseline HR data. For the experimental task, ECG task segments with more than one peak missing were excluded, and as before, participants with $\geq 50 \%$ missing task data were excluded $(n=1)$, leaving a final sample of $n=47$ for the frustration task HR data.

\section{Statistical Analysis}

All analyses were conducted in Stata 16. First, bivariate correlations were run between the ARI and demographic characteristics (age, sex, IQ and autism severity), and other measures of irritability (parentrated $A B C$ irritability subscale and the number of ODD symptoms from the CAPA), to confirm the construct validity of the ARI. Next, multilevel mixed-effect regression was used to test associations between ARI and trajectories of behavioural and physiological responses during the frustration task. The key term of interest was the time-by-irritability interaction, but we also tested for main effects of irritability, equating to an association with the overall number of presses/HR (rather than the slope of change). Any significant interactions were explored graphically using Empirical Bayes' estimates of behavioural and physiological response and low vs. high irritability, defined using a median split on the ARI. Age was included as a covariate in all task analyses, along with baseline physiology in HR analyses. As the behavioural data was the count of presses during each delay trial, a negative binomial model was specified. Likelihood ratio (LR) tests suggested a model with random intercept and slope was adequate for HR data, but the addition of a quadratic term of time (time ${ }^{2}$ ) was necessary for the press data (LR $\chi 2(1)=18.21, p<.01)$. After primary analyses, the following variables were added as covariates; IQ, autism severity, and medication status (coded as a binary variable of currently taking medication yes/no; split $45 / 55 \%(n=23 / 28)$; made up of $13 \%(n=3)$ minor tranquilizers/sedatives, $22 \%(n=5)$ stimulants, $4 \%(n=1)$ non-stimulants (e.g, atomoxetine, guanfacine, clonidine), 17\% $(n=4)$ anti-depressant, $9 \%(n=2)$ anticonvulsant, $22 \%(n=5)$ asthma medication, $52 \%(n=12)$ other medication) to assess the evidence for 
potential confounders (especially those which may index difficulties in understanding the task) on motor and physiological response. We report unstandardized coefficients throughout (b).

\section{Results}

Table 2 presents the bivariate correlations between ARI and demographic characteristics and other measures of irritability. ARI was not significantly correlated with age, sex, IQ or autism severity (all ps $>$.18). As expected, ARI was significantly correlated with the $A B C$ Irritability subscale $(r=.78, p<.001)$ and the number of ODD symptoms on the CAPA $(r=.71, p<.001)$.

Table 2

Bivariate Correlations between Demographic Characteristics and Measures of Irritability

\begin{tabular}{|c|c|c|c|c|c|c|c|}
\hline & 1 & 2 & 3 & 4 & 5 & 6 & 7 \\
\hline ARI Total (1) & - & & & & & & \\
\hline Age (2) & -.11 & - & & & & & \\
\hline Sex (3) & -.06 & .04 & - & & & & \\
\hline IQ (4) & .17 & -.07 & -.03 & - & & & \\
\hline $\begin{array}{l}\text { Autism Severity (5) } \\
\text { (ADOS-CSS) }\end{array}$ & .05 & -.13 & -.14 & $-.30 *$ & - & & \\
\hline ABC Irritability (6) & $.78^{\star \star}$ & -.08 & .14 & -.15 & .18 & - & \\
\hline CAPA Number of ODD Symptoms (7) & $.71^{\star \star}$ & -.01 & -.06 & .19 & .14 & $.62 * \star$ & - \\
\hline $\begin{array}{l}{ }^{\star p}<.01,{ }^{*} \mathrm{p}<.001 \\
\text { ADOS-CSS indicates Autism Diagnost } \\
\text { Aberrant Behavior Checklist; ARI Affec } \\
\text { Assessment; ODD Oppositional Defian }\end{array}$ & $\begin{array}{l}\text { ervatior } \\
\text { eactivity } \\
\text { rder. }\end{array}$ & $\begin{array}{l}\text { Schedu } \\
\text { ndex; } C\end{array}$ & $\begin{array}{l}-2 \text { calib } \\
\text { PA Chilc }\end{array}$ & $\begin{array}{l}\text { ted seve } \\
\text { and Ado }\end{array}$ & $\begin{array}{l}\text { scc } \\
\text { cent }\end{array}$ & $\begin{array}{l}\mathrm{ABC} \\
\mathrm{sychiatric}\end{array}$ & \\
\hline
\end{tabular}

\section{Associations between Irritability and Response to Frustration}

\section{Behavioural Response}

The main effects of time (incident rate ratio $(I R R)=.95, p=.14)$, time ${ }^{2}(\operatorname{IRR}=1.01, p=.34)$, age $(\operatorname{IRR}=.94$, $p=.64)$, and ARI (IRR = .94, $p=.13$ ) were all non-significant predictors of number of presses. The time-byARI interaction was a marginal but non-significant predictor $(I R R=.98, p=.06)$. The time-by-ARI interaction term indicates for a one-point increase in irritability, one would expect increase in the rate of button presses by a factor of 0.98 (i.e. a decrease). This is illustrated Fig. 2a, where trajectories of button 
presses are split by irritability threshold, where it appears that the high irritability group showed less change in response over the course of the task. When IQ, autism severity and medication use were included as covariates, the time-by-ARI interaction term was significant (IRR $=.98, p=.02)$. Neither IQ (IRR $=1.01, p=.81)$ nor autism severity (IRR $=1.09, p=.07)$ were significant predictors. Medication use was significantly associated with number of presses (IRR $=.45, \mathrm{p}<.01)$.

\section{Physiological Response}

There were significant main effects of both baseline HR $(b=.73, p<.01)$ and time $(b=.95, p<.01)$ in predicting HR, but not ARI $(b=-.24, p=.28)$ or age $(b=-.22, p=.77)$. The time-by-ARI interaction $(b=-.10, p$ $=.04$ ) was a significant predictor of HR. As illustrated in Fig. 2b, those scoring above the ARI cut-off were characterised by a higher HR at the beginning of the task followed by a less steep slope of increase. The addition of IQ, autism symptoms and medication use led the time-by-irritability interaction term to become non-significant $(b=-.10, p=.10)$. Neither IQ $(b=-.03, p=.40)$, autism severity $(b=-.08, p=.79)$ or medication use $(b=3.20, p=.06)$ were significant predictors of HR, although the coefficient for medication use was marginal.

\section{Discussion}

Previous work in typically developing children has implicated an aberrant response to frustration as a driver of irritability $(9,10)$. In the current study, we investigated whether irritability was associated with response to frustration using multi-modal assessment of behavioural and physiological markers, in a well-characterized sample of autistic youth. Results showed individuals with higher levels of irritability were characterised by a damped pattern of behavioural and physiological response to frustration, as indicated by flatter slopes of change during the experimental task. These associations largely remained when adjusting for IQ, autism severity and medication use.

In the current study we tested the role of response to mild provocation, by adapting an existing task designed to elicit frustration to be suitable for young people with a range of cognitive ability. The task involved exposing participants to a short period of delay, and this delay meant they thought would obtain a lower number of points than most people their age, thus can be situated with the Research Domain Criteria construct of 'frustrative non-reward' $(38,39)$. The current task is also relevant to the field of emotion regulation, where recent reviews have noted the over-reliance on parent or self-report measures of response to stressors in autistic populations, which may lead to results which are in part due to shared method variance (24). We note here that the task was completed by autistic youth with a wide range of functioning (IQ range of 33-129), and adjusting for IQ did not change the pattern of results, suggesting this experimental paradigm is likely applicable to more representative autistic samples than are typically included in experimental research. Previous work has used experimental paradigms to test associations between irritability and response to stress in autistic youth $(19,40)$, however, frustration and stress elicit different biological responses (20), and thus the relevance of each domain to irritability in autistic youth should be studied independently. We had hypothesised that individuals with irritability would show a greater response to frustration, however from inspection of plots of button presses over the course of the 
task (Fig. 2a), it appears that participants with higher levels of irritability did not show an increase in the number of button presses over time (unlike those with lower levels of irritability). This is contrary to our predictions, as previous research reports young adults with higher levels of attention-deficit hyperactivity disorder (ADHD) traits press the button more often on the delay trials (34) and children with ADHD find delay tasks more aversive (41), and a substantial proportion of young people with ADHD also have high levels of irritability (42). Therefore, we hypothesised that participants with irritability would find the delay more frustrating and press the button more when the computer became unresponsive. One interpretation is that a less steep slope of behavioural response reflects disengagement in the face of early frustration; participants with higher levels of irritability may have been less willing to continue to try and move the trials on when they appeared to get stuck. We also presented the frustration task at the end of a battery of other experimental tasks, which could have contributed to disengagement. Differences in task presentation may also be important to consider; compared to the original version of the task, we used simplified stimuli (selecting the smallest square from a choice of three) to allow for maximal participation. This may have led to a less engaging task for participants with higher IQ, so leading to less intense feelings of frustration, although this cannot explain the association with irritability. Further work combining objective behavioural measures such as button presses with observational coding of behaviour and subjective reports of mood is needed to clarify the meaning of these behavioural metrics.

In addition to associations with behavioural response, irritability was also associated with a different pattern of physiological response (as measured by change in HR) to frustration over the course of the task. Contrary to our predictions, results suggested that higher levels of irritability were associated with a flatter HR slope across the task. This blunted response is similar to findings from populations of typically developing youth characterised by high levels of irritability, mainly youth with disruptive behaviour disorders (e.g., ODD, conduct disorder), who exhibit a blunted physiological response to frustration (14, 15). Similarly, general population studies find high levels of trait aggression are associated with decreased neural response to experimentally induced frustration, especially in the frontal and limbic regions (17). Interestingly, previous work has also found irritability is associated with a blunted cortisol and HR response to stress in autistic samples of a similar age (19), and this effect was largely accounted for by co-occurring anxiety. In typically developing populations, a blunted response to frustration has been interpreted as indicative of an under-active fear system, which could lead to impairments in fearconditioning, stimulation seeking and risk-taking behaviours. We highlight that comparisons should be made with caution as the studies cited above did not measure irritability, but are useful to consider because they have studied groups with symptoms that are very similar to the behavioural manifestation of irritability in autistic populations (e.g., oppositionality, aggression, severe non-compliance).

A different interpretation is that the blunted response in those with high irritability in the current study may have been due to ceiling effects, in that those participants with high levels of irritability already had a higher HR at the beginning of the task (with the first measurement being the first delay trial). Indeed, from inspection of Fig. 2b, it appears that the high irritability group had a higher HR at the beginning of the task. Thus, it could be that in participants with high levels of irritability, fewer delay trial were required to increase HR, whereas in participants with lower levels of irritability, greater exposure to frustrating 
delays was required to elicit an increase in HR. This interpretation is in line with reports that HR reactivity is associated with conduct problems in children (18), and that higher HR precedes episodes of challenging behaviours in young children with ASD (22). Subtyping the types of behavioural difficulties under study may be key to understanding mixed findings: higher arousal is related to reactive aggression and anxiety, whereas lower arousal is more often associated with proactive aggression in typically developing youth (43). The construct of irritability is thought to encapsulate reactive outbursts rather than proactive acts (3), therefore the interpretation of hyper-reactivity in response to frustration is in keeping with this proposed division. Further work is needed to better characterise the nature of irritability and its behavioural manifestations in autistic vs. typically developing populations, in order to facilitate comparisons of aetiological mechanisms between the two groups.

\section{Strengths and Limitations}

To our knowledge this is one of the few studies to collect objective measurements of frustrative response in autistic youth. We explicitly designed the experimental task and selected the parent-rated measures to answer the question of whether irritability is associated with aberrant frustration response in autistic youth; the current paper presents a succinct test of this pre-specified hypothesis. Although the task has not been used in autistic populations before (but is often used in ADHD populations), the significant increase in HR over the course of the task and associations between both button press and HR and parent-rated irritability suggests the task was evoking frustration as designed to. Furthermore, the task was completed by a sample with a wide range of IQ, with minimal data loss, and results were largely unchanged when adjusting for IQ, severity of autism symptoms and medication use, suggesting the task may be appropriate for heterogenous samples of autistic youth. Given that reviews of the field have noted that research on emotion regulation and reactivity in autistic youth relies on questionnaire data (24), where parent-report measures may not fully capture emotional response, and self-report measures are not appropriate for very young children, or individuals who are minimally verbal and/or have significant levels of alexithymia, the development of valid experimental measures is an important goal. We also highlight our statistical approach; modelling change over time in the frustration task indicated that autistic individuals with irritability are characterised by a different trajectory of behavioural and physiological response to frustration. Averaging across all trials would have likely missed these differences that may be key to understanding the neurobiological basis of irritability. Additionally, the current sample was population-based (as opposed to samples recruited through current clinical attendance, which are known to be enriched for symptom severity), thus making it more representative of autism as a whole, and all participants had their diagnosis confirmed with 'gold-standard' diagnostic instruments. Although the subsample that completed the experimental task had a higher IQ than the full community-based sample, in all other key variables there were no significant differences. In terms of limitations, the lack of control group means whether a similar pattern of associations between cognition and behaviour are found in typically developing samples cannot be tested. We also acknowledge use of a moderately sized sample, which could have led to limited power to detect associations of smaller effect. 


\section{Conclusions}

Current results suggest that a maladaptive response to frustration may be one mechanism that underpins irritability and its behavioural manifestations (e.g., aggression, temper tantrums, challenging behaviours) in autistic populations. Further clarification, using multi-modal methodologies (e.g., observational and direct measurements paired with questionnaires), is required as to better understand whether irritability in autistic youth is characterised by hypo- or hyper-responsiveness to frustrating situations. This in turn will guide targets for future interventions, for example cognitive reappraisal and relaxation techniques (44). Given the poor outcomes for youth with severe irritability (1), it is imperative to understand the mechanisms by which autistic individuals develop irritability, and therefore how best to intervene. This paper presents an objective measure of frustration response and emotion regulation for use with autistic populations, and therefore is an important step towards this mechanistic understanding.

\section{List Of Abbreviations}

ABC Aberrant Behavior Checklist

ADHD attention deficit hyperactivity disorder

ADI-R Autism Diagnostic Interview - Revised

ADOS-CSS Autism Diagnostic Observation Schedule-2 Calibrated Severity Score

ARI Affective Reactivity Index

CAPA Child and Adolescent Psychiatric Assessment

DMDD disruptive mood dysregulation disorder

ECG electrocardiogram

HR heart rate

LR likelihood ratio

IQ intellectual quotient

IRR incident rate ratio

ODD oppositional defiant disorder

\section{Declarations}

Acknowledgements 
We would like to thank all the participants and their caregivers for taking the time to contribute to this study. We also thank Drs Jacqueline Bold and Mark O'Leary for assistance with the QUEST follow-up study.

\section{Author contributions}

VCL contributed to the design of the study, analysed data, wrote and edited the manuscript. GF collected data and helped to draft the manuscript. SC, IY and PW contributed to the design of the study, collected data, and helped to draft the manuscript. AP, TC and ES conceived of the study and helped to draft the manuscript. All authors read and approved the final manuscript.

\section{Ethics approval and consent to participate}

The study was approved by Camden and King's Cross Ethics Sub-Committee (17/LO/2098 for Wave 2, 17/LO/0397 for Wave 3 of QUEST study). All participating families gave their written informed consent (from young people themselves if $\geq 16$ years in age and were deemed to have capacity, otherwise from parents or caregivers).

\section{Consent for publication}

Not applicable.

\section{Availability of data and materials}

The datasets used and/or analysed during the current study are available on reasonable request.

\section{Competing Interests}

The authors declare they have no competing interests.

\section{Funding}

The original QUEST sample was funded by Clothworkers' Foundation, brokered by Research Autism (R011217 Autism M10 2011/12). The IAMHealth research programme was funded by the National Institute for Health Research (NIHR) under its Programme Grants for Applied Research programme (ES: RP-PG-1211-20016). The authors also acknowledge funding from NIHR Senior Investigator Awards (ES: NF-SI-0514-10073, AP: NF-SI-0617-10120), and a Sir Henry Wellcome Postdoctoral Fellowship (VCL: $213608 / Z / 18 / Z)$. The study was partially supported through the NIHR Maudsley Biomedical Research Centre at the South London and Maudsley NHS Foundation Trust in partnership with King's College London. The views expressed are those of the author(s) and not necessarily those of the NHS, the MRC, the NIHR or the Department of Health.

\section{References}


1. Vidal-Ribas P, Brotman MA, Valdivieso I, Leibenluft E, Stringaris A. The Status of Irritability in Psychiatry: A Conceptual and Quantitative Review. Journal of the American Academy of Child and Adolescent Psychiatry. 2016;55(7):556-70.

2. American Psychological Association. Diagnostic and Statistical Manual of Mental Disorders. 5th Edition. Arlington, VA.: American Psychiatric Publishing; 2013.

3. Leibenluft E. Severe mood dysregulation, irritability, and the diagnostic boundaries of bipolar disorder in youths. The American journal of psychiatry. 2011;168(2):129-42.

4. Mayes SD, Calhoun SL, Murray MJ, Ahuja M, Smith LA. Anxiety, depression, and irritability in children with autism relative to other neuropsychiatric disorders and typical development. Research in Autism Spectrum Disorders. 2011;5(1):474-85.

5. Simonoff E, Jones CRG, Pickles A, Happé F, Baird G, Charman T. Severe mood problems in adolescents with autism spectrum disorder. Journal of Child Psychology and Psychiatry. 2012;53(11):1157-66.

6. Mandy W, Roughan L, Skuse D. Three Dimensions of Oppositionality in Autism Spectrum Disorder. Journal of Abnormal Child Psychology. 2014;42(2):291-300.

7. Gadow KD, Devincent CJ, Pomeroy J, Azizian A. Comparison of DSM-IV symptoms in elementary school-age children with PDD versus clinic and community samples. Autism. 2005;9(4):392-415.

8. Maskey M, Warnell F, Parr JR, Le Couteur A, McConachie H. Emotional and Behavioural Problems in Children with Autism Spectrum Disorder. Journal of Autism and Developmental Disorders. 2013;43(4):851-9.

9. Leibenluft E. Pediatric Irritability: A Systems Neuroscience Approach. Trends in Cognitive Sciences. 2017;21(4):277-89.

10. Leibenluft E, Stoddard J. The developmental psychopathology of irritability. Development and psychopathology. 2013;25(4 Pt 2):1473-87.

11. Rich BA, Mariana S, Perez-Edgar KE, Fox N, Pine D, Leibenluft E. Different Psychophysiological and Behavioral Responses Elicited by Frustration in Pediatric Bipolar Disorder and Severe Mood Dysregulation. American Journal of Psychiatry. 2007;164(2):309-17.

12. Deveney CM, Connolly M, Haring CT, Bones BL, Reynolds RC, Kim P, et al. Neural Mechanisms of Frustration in Chronically Irritable Children. American Journal of Psychiatry. 2013;170(10):1186-94.

13. Perlman SB, Jones BM, Wakschlag LS, Axelson D, Birmaher B, Phillips ML. Neural substrates of child irritability in typically developing and psychiatric populations. Developmental Cognitive Neuroscience. 2015;14:71-80.

14. Fairchild G, van Goozen SHM, Stollery SJ, Brown J, Gardiner J, Herbert J, et al. Cortisol Diurnal Rhythm and Stress Reactivity in Male Adolescents with Early-Onset or Adolescence-Onset Conduct Disorder. Biological Psychiatry. 2008;64(7):599-606.

15. Northover C, Thapar A, Langley K, Fairchild G, van Goozen SHM. Cortisol levels at baseline and under stress in adolescent males with attention-deficit hyperactivity disorder, with or without comorbid conduct disorder. Psychiatry Research. 2016;242:130-6. 
16. Schoorl J, van Rijn S, de Wied M, van Goozen SHM, Swaab H. Neurobiological stress responses predict aggression in boys with oppositional defiant disorder/conduct disorder: a 1-year follow-up intervention study. European Child \& Adolescent Psychiatry. 2017;26(7):805-13.

17. Pawliczek CM, Derntl B, Kellermann T, Gur RC, Schneider F, Habel U. Anger under Control: Neural Correlates of Frustration as a Function of Trait Aggression. PLOS ONE. 2013;8(10):e78503.

18. Lorber MF. Psychophysiology of aggression, psychopathy, and conduct problems: a meta-analysis. Psychological bulletin. 2004;130(4):531.

19. Mikita N, Hollocks MJ, Papadopoulos AS, Aslani A, Harrison S, Leibenluft E, et al. Irritability in boys with autism spectrum disorders: an investigation of physiological reactivity. Journal of Child Psychology and Psychiatry. 2015;56(10):1118-26.

20. Moons WG, Eisenberger NI, Taylor SE. Anger and fear responses to stress have different biological profiles. Brain, Behavior, and Immunity. 2010;24(2):215-9.

21. Northrup JB, Goodwin M, Montrenes J, Vezzoli J, Golt J, Peura CB, et al. Observed emotional reactivity in response to frustration tasks in psychiatrically hospitalized youth with autism spectrum disorder. Autism. 2020;0(0):1-15.

22. Nuske HJ, Finkel E, Hedley D, Parma V, Tomczuk L, Pellecchia M, et al. Heart rate increase predicts challenging behavior episodes in preschoolers with autism. Stress. 2019;22(3):303-11.

23. Berkovits L, Eisenhower A, Blacher J. Emotion regulation in young children with autism spectrum disorders. Journal of Autism and Developmental Disorders. 2017;47(1):68-79.

24. Cai RY, Richdale AL, Uljarević M, Dissanayake C, Samson AC. Emotion regulation in autism spectrum disorder: Where we are and where we need to go. Autism Research. 2018;11(7):962-78.

25. Salazar F, Baird G, Chandler S, Tseng E, O'sullivan T, Howlin P, et al. Co-occurring psychiatric disorders in preschool and elementary school-aged children with autism spectrum disorder. Journal of autism and developmental disorders. 2015;45(8):2283-94.

26. Lord C, Rutter M, DiLavore P, Risi S, Gotham K, Bishop S. Autism diagnostic observation schedule second edition (ADOS-2). Torrance: Western Psychological Services; 2012.

27. Rutter M, Le Couteur A, Lord C. The Autism Diagnostic Interview-Revised: Western Psychological Services, Los Angeles; 2003.

28. Angold A, Costello EJ. The Child and Adolescent Psychiatric Assessment (CAPA). Journal of the American Academy of Child \& Adolescent Psychiatry. 2000;39(1):39-48.

29. Angold A, Prendergast M, Cox A, Harrington R, Simonoff E, Rutter M. The Child and Adolescent Psychiatric Assessment (CAPA). Psychological Medicine. 1995;25(4):739-53.

30. Stringaris A, Goodman R, Ferdinando S, Razdan V, Muhrer E, Leibenluft E, et al. The Affective Reactivity Index: a concise irritability scale for clinical and research settings. Journal of Child Psychology and Psychiatry. 2012;53(11):1109-17.

31. Aman M, Singh N. The Aberrant Behavior Checklist-Community. East Aurora, NY: Slosson Education Publications, Inc; 1994. 
32. Aman MG, Novotny S, Samango-Sprouse C, Lecavalier L, Leonard E, Gadow KD, et al. Outcome measures for clinical drug trials in autism. CNS Spectr. 2004;9(1):36-47.

33. Tools PS. E-Prime. Pittsburgh, PA: Psychology Software Tools; 2002.

34. Bitsakou P, Antrop I, Wiersema JR, Sonuga-Barke EJS. Probing the limits of delay intolerance: Preliminary young adult data from the Delay Frustration Task (DeFT). Journal of Neuroscience Methods. 2006;151(1):38-44.

35. Wilbertz G, Trueg A, Sonuga-Barke EJS, Blechert J, Philipsen A, Tebartz van Elst L. Neural and psychophysiological markers of delay aversion in attention-deficit hyperactivity disorder. Journal of Abnormal Psychology. 2013;122(2):566-72.

36. BIOPAC Systems I. AcqKnowledge 5.0.1. California: BIOPAC Systems, Inc; 2016.

37. Bazelmans T, Jones EJH, Ghods S, Corrigan S, Toth K, Charman T, et al. Heart rate mean and variability as a biomarker for phenotypic variation in preschoolers with autism spectrum disorder. Autism Research. 2019;12(1):39-52.

38. Insel T, Cuthbert B, Garvey M, Heinssen R, Pine DS, Quinn K, et al. Research domain criteria (RDoC): toward a new classification framework for research on mental disorders. American Journal of Psychiatry. 2010;167(7):748-51.

39. National Institute of Mental Health. Research Domain Criteria Matrix. 2020. https://www.nimh.nih.gov/research/research-funded-by-nimh/rdoc/constructs/rdoc-matrix.shtml. Accessed $2^{\text {nd }}$ October 2020.

40. Hollocks MJ, Pickles A, Howlin P, Simonoff E. Dual Cognitive and Biological Correlates of Anxiety in Autism Spectrum Disorders. Journal of Autism and Developmental Disorders. 2016;46(10):3295-307.

41. Sonuga-Barke E, Bitsakou P, Thompson M. Beyond the Dual Pathway Model: Evidence for the Dissociation of Timing, Inhibitory, and Delay-Related Impairments in Attention-Deficit/Hyperactivity Disorder. Journal of the American Academy of Child \& Adolescent Psychiatry. 2010;49(4):345-55.

42. Stringaris A, Goodman R. Three dimensions of oppositionality in youth. Journal of Child Psychology and Psychiatry. 2009;50(3):216-23.

43. Schoorl J, Van Rijn S, De Wied M, Van Goozen SHM, Swaab H. Variability in emotional/behavioral problems in boys with oppositional defiant disorder or conduct disorder: the role of arousal. European Child \& Adolescent Psychiatry. 2016;25(8):821-30.

44. Lochman J, Barry T, Pardini D. Anger control training for aggressive youth. In: Kazdin AE, Weisz JR, editors. Evidence-Based Psychotherapies for Children and Adolescents. New York: Guildford Press; 2003. p. 263-81.

\section{Figures}




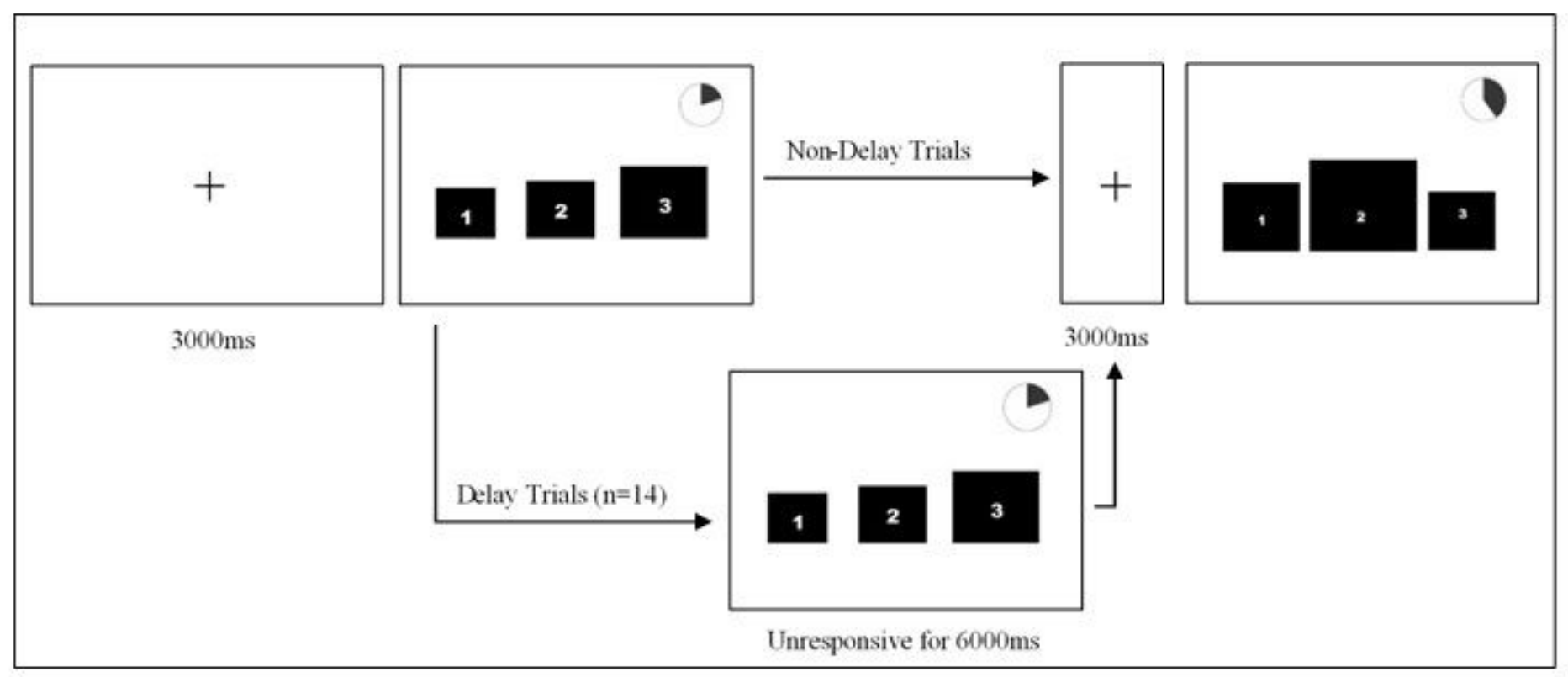

Figure 1

Schematic of Frustration Task

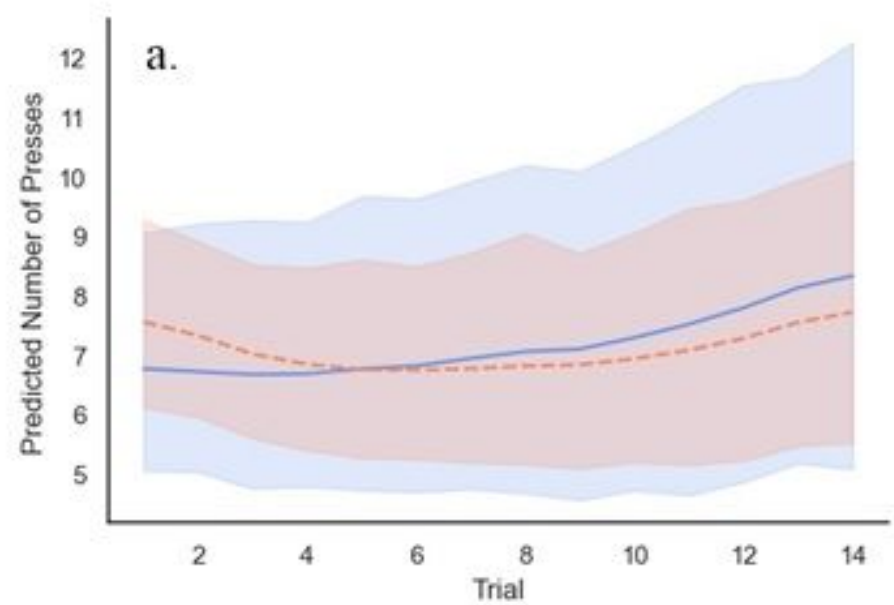

Levels of Irritability Based on Median Split

— Low Irritability

--- High Irritability

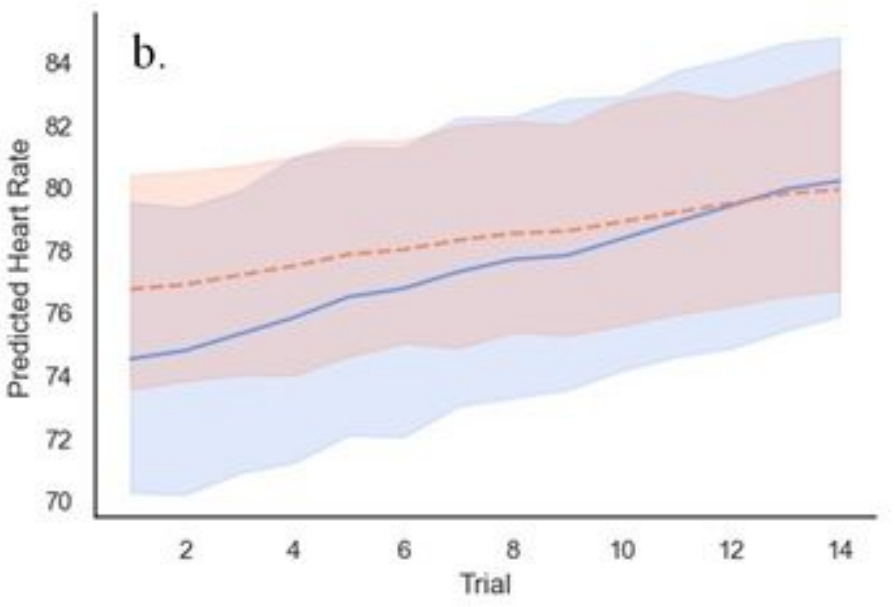

Levels of Irritability Based on Median Split

- Low Irritability

--- High Irritability 
Figure 2

Empirical Bayes' Estimates of a) Behavioural and b) Physiological Response to Frustration Over Course of the Task, Split by Level of Irritability 